\title{
mRNA export: threading the needle
}

\section{Ouassila Gaouar and Hugo Germain*}

Groupe de Recherche en Biologie Végétale, Département de Chimie et Physique, Université du Québec à Trois-Rivières, Trois-Rivières, OC, Canada

\author{
Edited by: \\ Susana Rivas, Laboratoire des \\ Interactions \\ Plantes-Microorganismes; Centre \\ National de la Recherche Scientifique, \\ France

\section{Reviewed by:} \\ Kemal Kazan, Commonwealth \\ Scientific and Industrial Research \\ Organization, Australia \\ Steven H. Spoel, University of \\ Edinburgh, UK \\ Xin Li, University of British Columbia, \\ Canada

\section{*Correspondence:} \\ Hugo Germain, Groupe de Recherche \\ en Biologie Végétale, Département de \\ Chimie et Physique, Université $d u$ \\ Québec à Trois-Rivières, 3351, \\ Boulevard des Forges, Trois-Rivières, \\ OC, Canada G9A 5 H7. \\ e-mail: hugo.germain@uqtr.ca
}

After mRNA biogenesis, several proteins interact with the messenger to ensure its proper export to the cytoplasm. Some of these proteins will bind RNA early on, at the onset of transcription by RNA polymerase II holoenzyme, while others will join later for downstream processing steps, such as poly-adenylation or splicing, or may direct mRNA ribonucleoprotein particle migration to the nucleopore. We recently discovered that Arabidopsis plant knockout for the protein MOS11 (MODIFIER OF $\underline{S} N C 1,11$ ) partially suppresses autoimmune responses observed in the TNL-type [TIR/NBS/LRR (Toll-interleukin-like receptor/nucleotide-binding site/C-terminal leucine-rich repeat)] R gene gain-of-function variant snc1 (suppressor of npr1-1, constitutive 1). This suppression of resistance to pathogens appears to be caused by a decrease in nuclear mRNA export in mos11-1 snc1 plants. In humans, the putative ortholog of MOS11, CIP29 (29-kDa cytokine-induced protein), interacts with three proteins that are also involved in mRNA export: DDX39 (DEAD-box RNA helicase), TAF15 of the FUS family (FUSED IN SARCOMA), and ALY (ALWAYS EARLY), a protein implicated in mRNA export in mammalian systems. These proteins have received very little attention in plants. Here, we will discuss their particularities and role in mRNA export and biotic stress.

Keywords: mRNA export, ALY, MOS11, CIP29, TAF15b, TAFs, RNA helicase, snc1

\section{INTRODUCTION}

Messenger RNA export is a tightly regulated process, unique to eukaryotes, that enhances control over the timing and level of translation. Albeit mRNA export is unidirectional, unlike nucleocytoplasmic protein trafficking, it represents a more complex process. Before mRNA can actually be led through the conduit formed by the nucleopore (NP) across the nuclear envelope (NE), it must be adequately processed and guided toward the NP basket. As mRNA elongates, it is loaded with proteins; some of them will mark intron-exon junctions, helicases will keep mRNA unwound, the spliceosome complex will bind to single-stranded nucleic acid, some will induce mRNA cleavage in its $3^{\prime}$ UTR so that it can then be $3^{\prime}$-poly-adenylated and bound with more proteins that recognize the poly-A tail. Additional proteins will serve as guides to direct mRNA to the NP. This association of proteins and one mRNA molecule is known as the messenger ribonucleoprotein particle (mRNP). In fact, mRNP thickness has been evaluated to be approximately 5-7 $\mathrm{nm}$ (Batisse et al., 2009). In comparison, the free, double-stranded DNA molecule has a width of $2 \mathrm{~nm}$, which increases to $10 \mathrm{~nm}$ when DNA is bound to nucleosomes. Hence, the traditional representation of a free and linear mRNA molecule generally illustrated in textbooks does not depict reality; rather mRNP is virtually the size of the double-stranded DNA molecule bound to nucleosomes.

Messenger ribonucleoprotein particle does not journey from the nucleoplasm to the cytoplasm by linear progression. As Siebrasse et al. (2012) beautifully demonstrated recently by light sheet microscopy, bottleneck of mRNP export occurs on the nuclear side of the NE, more precisely at the NP basket. Accumulation of $\mathrm{mRNP}$ at this precise location is indicative of intensive
mRNP remodeling required to remove mRNP nuclear proteins that will not engage in NP conduit along with mRNA during its migration toward the cytoplasm. The bulk of mRNP is exported by proteins of THO/TREX (transcription-export) complex (Strasser et al., 2002). However, the unidirectionality of transport is conferred, on the cytoplasmic side, by a RNA helicase that unloads the mRNP of key transport-related proteins, preventing its return to the nucleus (Tran etal., 2007; Stewart, 2010). TREX complex is highly conserved across species and orchestrates several steps between mRNA synthesis and export (Katahira, 2012).

Knowledge of mRNA export has been garnered in mammalian species, Drosophila and yeast. However, this crucial aspect of cellular biology and translation regulation has received very little attention in plants. Genetic screens designed to modify the autoimmune phenotype in the TNL-type [TIR-NBS-LRR (Toll-interleukin-like receptor/nucleotide-binding site/C-terminal leucine-rich repeat)] R gene gain-of-function variant sncl (suppressor of npr1-1, constitutive 1) have identified a number of proteins involved in nucleocytoplasmic transport (reviewed in Monaghan et al., 2010). MOS2, discovered in a sncl modifier screen, is the first protein potentially involved in mRNA export (Zhang et al., 2005). It encodes a nuclear protein of unknown function that possesses a RNA-binding domain. The G-patch found in MOS2 is also observed in eukaryotic RNA-processing proteins. The single mos2 mutant displays enhanced disease susceptibility to Pseudomonas syringae maculicola (P.s.m.) and Avr-containing Pseudomonas strains, indicating that MOS2 is involved in both basal and effector-triggered immunity (Zhang et al., 2005). Interestingly, MOS2 was recently found to be sumoylated (Miller et al., 2010). Sumoylation appears to be a functional regulatory key of 
proteins involved in mRNA export and processing (Meier, 2012). A number of studies targeting SUMO-enriched fraction or yeast two-hybrid with SUMO E2 or SUMO protease EARLY IN SHORT DAYS4 (ESD4) as baits discerned a significant number of proteins involved in RNA splicing and processing, DEAD/DEAH-box RNA helicases, and proteins with RNA-recognition motifs (Budhiraja et al., 2009; Elrouby and Coupland, 2010; Miller et al., 2010). MOS3 (also called SAR3/AtNup96), another protein discovered in snc1 modifier screen (Zhang and Li, 2005), is an integral NP component of the conserved Nup107-Nup160 complex shown to be required for mRNA export (Parry et al., 2006). Similarly to mos2, single mos3 mutant plants display enhanced disease susceptibility to P.s.m. and Avr-containing Pseudomonas strains linking MOS3 to both basal and effector-triggered immunity (Zhang and Li, 2005).

We recently reported the discovery of a novel $s n c 1$ modifier,

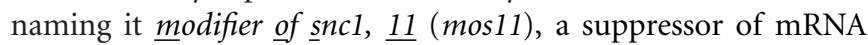
export (Germain et al., 2010). Unlike most previously identified mos mutants, including mos 2 and mos3, single mos 11 mutant does not display enhanced disease susceptibility, indicating that its role in immunity, observed in suppression of the snc1 autoimmune phenotype, may be limited to $\mathrm{R}$ gene-mediated resistance. Although cellular function of the putative homolog of MOS11 in mammalian systems (CIP29, 29-kDa cytokine-induced protein) was not established, the physical interactors encountered in human cells are consistent with a role in mRNA export. In this review, we focus on MOS11 and interactors of the homolog of MOS11, found in non-plant systems. Since none of them has been described in plants, we will hypothesize their putative involvement in mRNA export and innate plant immunity.

\section{MOS11}

mos11 mutant was initially identified by T-DNA tagging in the sncl background. Typically, sncl plants are dwarfs that have elevated salicylic acid levels, constitutive pathogenesis-related genes expression and enhanced resistance to Hyaloperonospora arabidopsidis Noco 2 and virulent Pseudomonas syringae strains. mos11 snc1 plants do not fully revert to wild type-looking plants; mos 11 only partially suppresses the molecular and morphological features of sncl mutants. With inverse polymerase chain reaction, we identified a T-DNA insertion in At5g02770 and confirmed, by complementation with the wild type At5g02770 sequence, that this T-DNA insertion was causing the mos11 sncl phenotype (Germain etal., 2010). At5g02770 encodes a small protein of unknown function, and its sequence is unique in Arabidopsis. The human protein CIP29 (Table 1) is the closest homolog with a putative function found with BlastP (Fukuda et al., 2002). Insight into its putative role came with the identification of two yeast twohybrid interactors of CIP29: the DEAD-box RNA helicases BAT1 and DDX39 (DEAD-box RNA helicase; Leaw et al., 2004). Patches of positively charged residues in CIP29 (and MOS11) indicate affinity for DNA or RNA and in vitro assays have demonstrated that CIP29 binds RNA (Sugiura et al., 2007). The observation that the RNA helicase activity of DDX39 was greatly enhanced in the presence of CIP29 provided a molecular role for CIP29 (Sugiura et al., 2007). In addition, these authors demonstrated that DDX39 could immunoprecipitate both CIP29 and ALY (ALWAYS EARLY). Dufu et al. (2010) established that recruitment of CIP29 to mRNA is capping- and splicing-dependent, positioning the role of CIP29 as a post-splicing event. Finally, the yeast ortholog of CIP29, Tho1 (Transcriptional defect of $\underline{H}$ pr 1 by overexpression), can suppress the RNA export defect of $h p r 1 \Delta$ when overexpressed (Jimeno et al., 2006). Using whole mount in situ total mRNA localization, we clearly determined that mos 11 and mos11 sncl plants manifest decreased mRNA export and accumulate poly-A mRNA in their nuclei (Germain et al., 2010). Surprisingly, despite a rather dramatic decrease in mRNA export, plant morphology appears to be virtually identical to that of wild type plants, indicating that all mRNA probably eventually reach the cytosol. The important transcriptional reprograming responsible for sncl elevated defense is likely to be dimmed in this mutant that shows nuclear mRNAs accumulation.

\section{TAF15B}

RNA polymerase II pre-initiation complex assembly requires the presence of general transcription factors (GTFs). Transcription factors for polymerase IID (TFIID), one of these GTFs, comprises a complex of several subunits that include TATA-box binding protein (TBP) and TBP-associated factors (TAFs). The combination of these proteins makes up the GTF TFIID, the major core promoter recognition factor that provides scaffolding for the assembly of other GTFs. TAFs can bind activators and other transcriptional regulators; some of them exert catalytic

Table 1 | CIP29 interacting proteins and their putative orthologs in Arabidopsis.

\begin{tabular}{lll}
\hline Human gene (NCBI gene ID) & $\begin{array}{l}\text { Arabidopsis ortholog } \\
\text { (TAIR gene ID) }\end{array}$ & Putative function
\end{tabular}

CIP29 (84324)

TAF15 (8148)

DDX39B/UAP56/BAT1 (7919)

ALY/REF/BEF/THOC4 (10189)

$\begin{array}{ll}\text { MOS11 (At5g02770) } & \text { Activation of DDX39 } \\ \text { AtTAF15b (At5g58470) } & \text { Unknown } \\ \text { AtRH15 (At5g11170) } & \text { DEAD-box RNA helicase } \\ \text { AtRH15b (At5g11200) } & \\ \text { AtALY1 (At5g59950) } & \text { TREX complex adaptor protein } \\ \text { AtALY2 (At5g02530) } & \\ \text { AtALY3 (At1g66260) } & \\ \text { AtALY4 (At5g37720) } & \end{array}$

MOS11 (At5g02770)

AtRH15 (At5g11170)

AtALY4 (At5g37720)
Sugiura et al. (2007), Germain et al. (2010)

Sugiura et al. (2007)

Aubourg et al. (1999)

Dufu et al. (2010)

Pendle et al. (2005)

Uhrig etal. (2004)

Yelina et al. (2010) 
activity modifying the histone code and transcriptional regulators. For example, TAF1 alone displays kinase, acetyltransferase, and ubiquitin-activating/conjugating enzyme activity, all in one protein. TAF1 can de-condense chromatin by $\mathrm{H} 3-\mathrm{H} 4$ histone acetylation and ubiquitination of histone $\mathrm{H} 1$; with its two kinase domains, it also directly phosphorylates some transcription factors (Dikstein et al., 1996; Mizzen et al., 1996; Pham and Sauer, 2000). Mining the Arabidopsis genome identified 18 putative TAFs, including TAF15a and TAF15b (Lago et al., 2004). AtTAF15b (Table 1) is the Arabidopsis homolog closest to the TAF protein that interacts in human cells with CIP29. Although some TAFs, e.g., TAF1, have been characterized in Arabidopsis (Bertrand et al., 2005; Benhamed etal., 2006), TAF15b has not. Since TAF15b does not contain any conserved catalytic domain that would provide insight into its putative molecular function, we investigated whether it has an effect on mRNA export. Whole mount in situ mRNA localization on two T-DNA lines (Salk_061974 and Sail_35_B06), inserted 17 bp apart in the third intron of At5g58470, did not reveal any significant changes in the amount of nuclear mRNA compared to wild type plants (unpublished). Thus, it is likely that TAF15b is not absolutely required for mRNA export under our assay conditions or that it is needed only for export of certain mRNAs, which is not possible to detect with poly-A mRNA localization. We are currently investigating the putative role of TAF15b in innate plant immunity and other cellular functions.

\section{DEAD-BOX RNA HELICASE}

Employing BlastP with CIP29-interacting RNA helicase, we identified two Arabidopsis proteins (At5g11170 and At5g11200) with identical e-values. Both helicases are separated by only two genes on chromosome 5 and show 100\% identity at the amino acid level; neither has been studied in plants. Previous mining of Arabidopsis sequences unveiled the presence of 32 DEAD-box RNA helicases in Arabidopsis (Aubourg et al., 1999). Based on the nomenclature proposed by them, At5g11170 is AtRH15 (Table 1). However, At5g11200 was not identified by Aubourg et al. (1999), perhaps due to its high degree of similarity to At5g11170. Therefore, we suggest re-naming At5g11200 as AtRH15b. The new TAIR (The Arabidopsis Information Resource) annotation identifies At5g11170 and At5g11200 as homologs of human UAP56. Alternate UAP56 names are DDX39 and BAT1, and we refer to UAP56 as DDX39 since it has been linked to CIP29 as the human DDX39. Enzymes that tap into energy released by the hydrolysis of a nucleotide triphosphate to unwind double-stranded RNAs are defined as RNA helicases (de la Cruz et al., 1999), linking them to every possible step in mRNA biogenesis and function. In the context of mRNA export, the unwinding activity of helicases can make mRNA more or less accessible to mRNA-processing proteins, thereby facilitating the addition and removal of proteins, and are involved in mRNP remodeling at the NP basket site. As mentioned previously, CIP29 is known to amplify the RNA-unwinding activity of DDX39 (Sugiura et al., 2007). The precise roles of AtRH15 and AtRH15b are unknown, and since their amino acid sequences are identical, it is likely that they complement each other functionally. It would be interesting to assess whether MOS11 can increase the RNA-unwinding activity of AtRH15 and/or AtRH15b as CIP29 does for DDX39. It should be mentioned that AtRH15 (like many helicases) can be sumoylated (Meier, 2012).

Very few functional analyses of DEAD-box RNA helicases have been conducted in Arabidopsis. Genetic screening of plants showing cold sensitivity identified LOS4 (LOW EXPRESSION OF OSMOTICALLY RESPONSIVE GENES4; Gong et al., 2002), which encodes a DEAD-box RNA helicase that localizes to the nucleus and cytoplasm. los4-1 mutant plants are very sensitive to chilling temperatures, particularly in the dark. LOS4 has also been shown to be required for mRNA export in a temperaturedependent manner, and LOS4-GFP (green fluorescent protein) fusion accumulates at the NE (Gong et al., 2002, 2005) where it may be involved in mRNP remodeling.

In human cells, the helicase DEAD-box protein 5 (Dbp5) is known to be required for the directionality of mRNA export (Tran et al., 2007). Dbp5 accumulates on the cytoplasmic side of the NE where it removes Nab2 from mRNP (Tran et al., 2007). Export functions as a cargo:carrier complexe; once mRNA has reached the cytosol, the carrier is disassembled to prevent cargo re-entry from the destination compartment (Stewart, 2010). The helicase that controls directionality in plants is unknown. However, LOS4 is the Arabidopsis helicase with the strongest sequence similarity to Dbp5; it accumulates at the nucleus outer membrane and is observed in the cytoplasm and nucleus (Gong et al., 2005). Two other helicases - STRS1 and STRS2 (STRESS RESPONSE SUPPRESSOR) corresponding to AtRH5 (At1g31970) and AtRH25 (At5g08620), respectively - have been shown to induce tolerance to salt, osmotic and heat stresses, suggesting that helicases suppress responses to abiotic stress (Kant et al., 2007). However, it is not known whether these helicases are involved in mRNA export. More than 30 helicases have been identified in Arabidopsis and it is conceivable that one helicase or a specific group of helicases may have specificity for groups of transcripts such as transcripts induced after biotic stress.

\section{ALY}

Counterparts of the metazoan $A L Y$ (also known as REF, BEF, and THOC4) and its Saccharomyces cerevisiae homolog YRA1 exist in plants (Table 1); as in animals, the number of ALY-coding genes in plants varies by species (Uhrig et al., 2004; Dufu et al., 2010). The exact functions of plant ALYs are not yet known. Four ALY genes have been reported so far in Arabidopsis thaliana: At5g59950, At5g02530, At1g66260, and At5g37720 (hereafter referred to as AtALY1, AtALY2, AtALY3, and AtALY4; Uhrig et al., 2004; Pendle et al., 2005; Yelina et al., 2010). Despite the relatively moderate sequence similarity between AtALYs (55-71\%), AtALY1, AtALY2, AtALY3, and AtALY4 respectively share 41, 42, 38, and 48\% sequence identity with human ALY. All four AtALYs localize to the nucleoplasm and, with the exception of AtALY2, also accumulate in the nucleolus, a poorly studied compartment in plants (Uhrig et al., 2004; Pendle et al., 2005; Shaw and Brown, 2012). Microarray data from Genevestigator (https://www.genevestigator.com/gv/) indicate medium to high expression levels for the four AtALY genes in seedlings, inflorescences, and shoots; lack of expression data for other tissues, however, makes it difficult to determine whether or not $A t A L Y$ gene expression is tissue-specific. 
Current models of mRNA export in animals and yeast depict ALY as a conserved component of TREX complex along with THO subcomplex, UAP56 (Sub2 in yeast), TEX1, and CIP29 (Tho1 in yeast), as a bona fide component (Carmody and Wente, 2009; Dufu et al., 2010; Chi et al., 2012; Katahira, 2012). However, recruitment of TREX complex components to mRNAs appears to proceed via distinct mechanisms in metazoans and yeast, possibly because of differences in gene structure, as the yeast genome contains far less introns than animal genomes (Cheng et al., 2006). Briefly, ALY and UAP56 are recruited to $\mathrm{mRNA}$ in an interdependent manner; UAP56 is then displaced by the Nxf1-Nxt1/TAP-p15 (Mex67Mtr2 in yeast) heterodimer which is involved in mRNP targeting of nuclear pore complex (NPC; Carmody and Wente, 2009; Dufu et al., 2010; Walsh et al., 2010; Chi etal., 2012; Katahira, 2012). In human cells, TREX complex is recruited mostly in a splicingand cap-dependent manner and binds near the $5^{\prime}$ end of spliced mRNA through interaction between ALY and the cap-binding complex protein CBP80. This mRNA $5^{\prime}$-end positioning of TREX complex is thought to account for the $5^{\prime}-3^{\prime}$ orientation of the mRNA molecule during its export (Cheng et al., 2006; Kohler and Hurt, 2007).

Experimental results on Arabidopsis support the hypothesis that THO/TREX complex is conserved not only in yeast and metazoans, but also in plants, and strongly indicate a role for this complex (or at least some of its components) in plant immunity (Furumizu et al., 2010; Yelina et al., 2010; Pan et al., 2012b). For example, AtTEX1 and AtTHO1 have been shown to be required for the biogenesis of a subset of trans-acting small interfering RNAs (tasiRNAs; Yelina et al., 2010). Interestingly, it is now known that, in Fabaceae and Solanaceae, tasiRNAs mediate nucleotidebinding leucine-rich repeat gene silencing in the absence of pathogen threats (Zhai et al., 2011; Li et al., 2012). Furthermore, AtHPR1, a component of THO subcomplex, was recently identified as a mediator of disease resistance. hprl-4 mutant plants display compromised mRNA export and lack basal resistance to virulent bacterial and fungal strains (Pan et al., 2012a). To our knowledge, HPR1 is the only free nuclear protein (not bound to the NE) known to affect both mRNA export and basal defense responses.

Arabidopsis and Nicotiana benthamiana ALY proteins have been found to interact with P19 silencing suppressor protein of Tomato bushy stunt virus (Uhrig et al., 2004; Canto et al., 2006). ALYP19 interaction results in the relocalization of AtALY2, AtALY4,

\section{REFERENCES}

Adams-Phillips, L., Briggs, A. G., and Bent, A. F. (2010). Disruption of poly(ADP-ribosyl)ation mechanisms alters responses of Arabidopsis to biotic stress. Plant Physiol. 152, 267-280.

Aubourg, S., Kreis, M., and Lecharny, A. (1999). The DEAD box RNA helicase family in Arabidopsis thaliana. Nucleic Acids Res. 27, 628-636.

Batisse, J., Batisse, C., Budd, A., Bottcher, B., and Hurt, E. (2009). Purification of nuclear poly(A)-binding protein Nab2

and two N. benthamiana ALY proteins from the nucleus to the cytoplasm; however, it also leads to accumulation of P19 in the nucleolus by those ALY proteins that do not re-localize to the cytoplasm (Uhrig etal., 2004; Canto et al., 2006). Interestingly, nucleolar targeting of P19 interferes with its silencing suppression activity (Canto et al., 2006). Unfortunately, the biological role of ALY-P19 interaction is still unknown.

Yeast two-hybrid and in vitro findings have raised the assumption that AtALY3 and AtALY4 likely interact with the nuclear enzyme poly(ADP-ribose) polymerase 1 (PARP1; Storozhenko et al., 2001). Although this interaction has not been demonstrated in planta, it is noteworthy that plant PARPs have been reported to mediate some of the immune responses triggered upon recognition of microbe-associated molecular patterns (Adams-Phillips et al., 2010).

In mammals, ALY is emerging as a versatile protein involved in processes other than mRNA export. Thus, it is thought to stabilize some viral transcripts independently of their export (Stubbs et al., 2012). Furthermore, misregulation of ALY expression has been associated with tumorigenesis (Dominguez-Sanchez et al., 2011). In plants, such versatility would be consistent with the multiplicity of $A L Y$ genes and the heterogeneity of ALY protein subnuclear distribution.

\section{CONCLUDING REMARKS}

This review highlights several similarities between the mechanistic aspects of animal and plant mRNA export processes. However, key questions remain to be answered that could promote understanding of plant mRNA export specificities. Which helicase is driving the directionality of export in plants? Does MOS11 possess helicase activity-enhancing capacity? What is the true role of the different ALYs, why are there four ALYs in plants, what role do they play in the nucleolus, can ALY bind suppressors of silencing other than P19? Plant nuclear proteome dynamics is still largely unknown, even more so the nuclear proteome of biotic or abiotic stressed plants. Although confocal imagery and genetics will remain core tools in resolving these issues, thorough proteomics analysis could lead geneticists on the right track. The fact that several pathogen virulence factors appear to be targeting the nucleus, combined with the observed high level of conservation of the mRNA export machinery suggest that the export machinery would represent a good target for pathogen effectors.

gene encoding TATA-binding protein (TBP)-associated factor TAF1, is required to integrate light signals to regulate gene expression and growth. J. Biol. Chem. 280, 1465 1473.

Budhiraja, R., Hermkes, R., Muller, S., Schmidt, J., Colby, T., Panigrahi, K., et al. (2009). Substrates related to chromatin and to RNA-dependent processes are modified by Arabidopsis SUMO isoforms that differ in a conserved residue with influence on desumoylation. Plant Physiol. 149, 1529-1540.
Canto, T., Uhrig, J. F., Swanson, M., Wright, K. M., and Macfarlane, S. A. (2006). Translocation of tomato bushy stunt virus P19 protein into the nucleus by ALY proteins compromises its silencing suppressor activity. J. Virol. 80, 9064-9072.

Carmody, S. R., and Wente, S. R. (2009). mRNA nuclear export at a glance. $J$. Cell Sci. 122, 1933-1937.

Cheng, H., Dufu, K., Lee, C. S., Hsu, J. L., Dias, A., and Reed, R. (2006). Human mRNA export machinery recruited to the $5^{\prime}$ end of mRNA. Cell 127, 1389-1400. 
Chi, B., Wang, Q., Wu, G., Tan, M., Wang, L., Shi, M., et al. (2012). Aly and THO are required for assembly of the human TREX complex and association of TREX components with the spliced mRNA. Nucleic Acids Res. 41, 1294-1306.

de la Cruz, J., Kressler, D., and Linder, P. (1999). Unwinding RNA in Saccharomyces cerevisiae: DEAD-box proteins and related families. Trends Biochem. Sci. 24, 192-198.

Dikstein, R., Ruppert, S., and Tjian, R. (1996). TAFII250 is a bipartite protein kinase that phosphorylates the base transcription factor RAP74. Cell 84, 781-790.

Dominguez-Sanchez, M. S., Saez, C., Japon, M. A., Aguilera, A., and Luna, R. (2011). Differential expression of THOC1 and ALY mRNP biogenesis/export factors in human cancers. BMC Cancer 11:77. doi: 10.1186/1471-2407-11-77

Dufu, K., Livingstone, M. J., Seebacher, J., Gygi, S. P., Wilson, S. A., and Reed, R. (2010). ATP is required for interactions between UAP56 and two conserved mRNA export proteins, Aly and CIP29, to assemble the TREX complex. Genes Dev. 24, 2043-2053.

Elrouby, N., and Coupland, G. (2010). Proteome-wide screens for small ubiquitin-like modifier (SUMO) substrates identify Arabidopsis proteins implicated in diverse biological processes. Proc. Natl. Acad. Sci. U.S.A. 107, 17415-17420.

Fukuda, S., Wu, D. W., Stark, K., and Pelus, L. M. (2002). Cloning and characterization of a proliferationassociated cytokine-inducible protein, CIP29. Biochem. Biophys. Res. Commun. 292, 593-600.

Furumizu, C., Tsukaya, H., and Komeda, Y. (2010). Characterization of EMU, the Arabidopsis homolog of the yeast THO complex member HPR1. RNA 16, 1809-1817.

Germain, H., Qu, N., Cheng, Y. T., Lee, E., Huang, Y., Dong, O. X., etal. (2010). MOS11: a new component in the mRNA export pathway. PLoS Genet. 6:e1001250. doi: 10.1371/journal.pgen. 1001250

Gong, Z., Dong, C. H., Lee, H., Zhu, J., Xiong, L., Gong, D., et al. (2005). A DEAD box RNA helicase is essential for mRNA export and important for development and stress responses in Arabidopsis. Plant Cell 17, 256-267.

Gong, Z., Lee, H., Xiong, L., Jagendorf, A., Stevenson, B., and Zhu, J. K. (2002). RNA helicase-like protein as an early regulator of transcription factors for plant chilling and freezing tolerance. Proc. Natl. Acad. Sci. U.S.A. 99, 11507-11512.

Jimeno, S., Luna, R., Garcia-Rubio, M. and Aguilera, A. (2006). Thol, a nove hnRNP, and Sub2 provide alternative pathways for mRNP biogenesis in yeast THO mutants. Mol. Cell. Biol. 26, 4387-4398.

Kant, P., Kant, S., Gordon, M., Shaked, R., and Barak, S. (2007). STRESS RESPONSE SUPPRESSOR1 and STRESS RESPONSE SUPPRESSOR2, two DEAD-box RNA helicases that attenuate Arabidopsis responses to multiple abiotic stresses. Plant Physiol. 145, 814-830.

Katahira, J. (2012). mRNA export and the TREX complex. Biochim. Biophys. Acta 1819, 507-513.

Kohler, A., and Hurt, E. (2007). Exporting RNA from the nucleus to the cytoplasm. Nat. Rev. Mol. Cell Biol. 8, 761-773.

Lago, C., Clerici, E., Mizzi, L. Colombo, L., and Kater, M. M. (2004). TBP-associated factors in Arabidopsis. Gene 342, 231-241.

Leaw, C. L., Ren, E. C., and Choong, M. L. (2004). Hcc-1 is a novel component of the nuclear matrix with growth inhibitory function. Cell. Mol. Life Sci. 61, 2264-2273.

Li, F., Pignatta, D., Bendix, C., Brunkard, J. O., Cohn, M. M., Tung, J., et al. (2012). MicroRNA regulation of plant innate immune receptors. Proc. Natl. Acad. Sci. U.S.A. 109, 1790-1795.

Meier, I. (2012). mRNA expor and sumoylation - lessons from plants. Biochim. Biophys. Acta 1819, 531-537.

Miller, M. J., Barrett-Wilt, G. A., Hua, Z., and Vierstra, R. D. (2010). Proteomic analyses identify a diverse array of nuclear processes affected by small ubiquitin-like modifier conjugation in Arabidopsis. Proc. Natl. Acad. Sci. U.S.A. 107, 16512-16517.

Mizzen, C. A., Yang, X. J., Kokubo T., Brownell, J. E., Bannister, A. J., Owen-Hughes, T., et al. (1996). The TAFII250 subunit of TFIID has histone acetyltransferase activity. Cell $87,1261-1270$

Monaghan, J., Germain, H., Weihmann, T., and Li, X. (2010). Dissecting plant defence signal transduction: modifier of sncl in Arabidopsis. Can. J. Plant Pathol. 32, 35-42.

Pan, H., Liu, S., and Tang, D. (2012a). The THO/TREX complex functions in disease resistance in Arabidopsis. Plant Signal. Behav. 7, 422-424.
Pan, H., Liu, S., and Tang, D. (2012b). HPR1, a component of the THO/TREX complex, plays an important role in disease resistance and senescence in Arabidopsis. Plant J. 69, 831-843.

Parry, G., Ward, S., Cernac, A., Dharmasiri, S., and Estelle, M. (2006). The Arabidopsis SUPPRESSOR OF AUXIN RESISTANCE proteins are nucleoporins with an important role in hormone signaling and development. Plant Cell 18, 1590-1603.

Pendle, A. F., Clark, G. P., Boon, R., Lewandowska, D., Lam, Y. W. Andersen, J., et al. (2005). Proteomic analysis of the Arabidopsis nucleolus suggests novel nucleolar functions. Mol. Biol. Cell 16, 260-269.

Pham, A. D., and Sauer, F. (2000). Ubiquitin-activating/conjugating activity of TAFII250, a mediator of activation of gene expression in Drosophila. Science 289, 2357-2360.

Shaw, P., and Brown, J. (2012). Nucleoli: composition, function, and dynamics. Plant Physiol. 158, 44-51.

Siebrasse, J. P., Kaminski, T., and Kubitscheck, U. (2012). Nuclear export of single native mRNA molecules observed by light sheet fluorescence microscopy. Proc. Natl. Acad. Sci. U.S.A. 109, 9426-9431.

Stewart, M. (2010). Nuclear export of mRNA. Trends Biochem. Sci. 35, 609-617.

Storozhenko, S., Inze, D., Van Montagu, M., and Kushnir, S. (2001). Arabidopsis coactivator ALY-like proteins, DIP1 and DIP2, interact physically with the DNA-binding domain of the Zn-finger poly(ADP-ribose) polymerase. J. Exp. Bot. 52, 13751380.

Strasser, K., Masuda, S., Mason, P., Pfannstiel, J., Oppizzi, M., Rodriguez-Navarro, S., et al. (2002). TREX is a conserved complex coupling transcription with messenger RNA export. Nature 417, 304-308.

Stubbs, S. H., Hunter, O. V., Hoover, A., and Conrad, N. K. (2012). Viral factors reveal a role for REF/Aly in nuclear RNA stability. Mol. Cell. Biol. 32, 1260-1270.

Sugiura, T., Sakurai, K., and Nagano, Y. (2007). Intracellular characterization of DDX39, a novel growth-associated RNA helicase. Exp. Cell Res. 313, 782-790.

Tran, E. J., Zhou, Y., Corbett, A. H., and Wente, S. R. (2007). The DEAD-box protein Dbp5 controls mRNA export by triggering specific RNA:protein remodeling events. Mol. Cell 28, 850-859.
Uhrig, J. F., Canto, T., Marshall, D., and Macfarlane, S. A. (2004). Relocalization of nuclear ALY proteins to the cytoplasm by the tomato bushy stunt virus P19 pathogenicity protein. Plant Physiol. 135, 2411-2423.

Walsh, M. J., Hautbergue, G. M., and Wilson, S. A. (2010). Structure and function of mRNA export adaptors. Biochem. Soc. Trans. 38, 232-236.

Yelina, N. E., Smith, L. M., Jones, A. M., Patel, K., Kelly, K. A., and Baulcombe, D. C. (2010). Putative Arabidopsis THO/TREX mRNA export complex is involved in transgene and endogenous siRNA biosynthesis. Proc. Natl. Acad. Sci. U.S.A. 107, 13948-13953.

Zhai, J., Jeong, D. H., De Paoli, E., Park, S., Rosen, B. D., Li, Y., et al. (2011). MicroRNAs as master regulators of the plant NB-LRR defense gene family via the production of phased, trans-acting siRNAs. Genes Dev. 25, 2540-2553.

Zhang, Y., Cheng, Y. T., Bi, D., Palma, K., and Li, X. (2005). MOS2, a protein containing G-patch and KOW motifs, is essential for innate immunity in Arabidopsis thaliana. Curr. Biol. 15, 1936-1942.

Zhang, Y., and Li, X. (2005). A putative nucleoporin 96 is required for both basal defense and constitutive resistance responses mediated by suppressor of npr1-1, constitutive 1. Plant Cell 17, 1306-1316.

Conflict of Interest Statement: The authors declare that the research was conducted in the absence of any commercial or financial relationships that could be construed as a potential conflict of interest.

Received: 20 January 2013; paper pending published: 06 February 2013; accepted: 04 March 2013; published online: 22 March 2013.

Citation: Gaouar $O$ and Germain $H$ (2013) mRNA export: threading the needle. Front. Plant Sci. 4:59. doi: 10.3389/ fpls.2013.00059

This article was submitted to Frontiers in Plant-Microbe Interaction, a specialty of Frontiers in Plant Science.

Copyright (c) 2013 Gaouar and Germain. This is an open-access article distributed under the terms of the Creative Commons Attribution License, which permits use, distribution and reproduction in other forums, provided the original authors and source are credited and subject to any copyright notices concerning any thirdparty graphics etc. 